\title{
Wird die alevitische Jugend das Alevitentum reformieren? - Eine Generation auf der Suche nach einer Neuformullierung
}

\author{
Martina Loth
}

\section{Die Ausgangssituation der Jugendlichen}

Das Alevitentum war in Deutschland bis vor einigen Jahrzehnten einer breiten Öffentlichkeit kaum bekannt. Die Alevitinnen und Aleviten hatten sich eher bedeckt gehalten und traten äußerst selten öffentlich auf. Anfang der 199oer Jahre begann jedoch ein Prozess, in dessen Verlauf die Glaubensgemeinschaft an Selbstbewusstsein und Anerkennung gewonnen hat. Eine wichtige Rolle spielte hierbei die Alevitische Gemeinde Deutschland e. V. (Almanya Alevi Birlikleri Federasyonu, $\mathrm{AABF})$, die der Dachverband der alevitischen Gemeinden in Deutschland ist. Seit kurzem lässt sich allerdings beobachten, dass der der AABF unterstehende Jugendverband, der Bund der Alevitischen Jugendlichen in Deutschland e.V. (BDAJ), sich in einer beachtlichen Entwicklung befindet: Er wird immer größer, einflussreicher und setzt einige andere Schwerpunkte in seiner Arbeit und seinem Auftreten als die AABF.

Diese Entwicklung geht vor allem darauf zurück, dass sich die jüngere Generation in einer anderen Ausgangssituation befindet, als die älteren Generationen. Die Jüngeren wachsen in einem Umfeld auf, in dem sie ihre alevitische Herkunft nicht mehr verstecken müssen. Dadurch ergeben sich einige bedeutende Unterschiede zwischen der jüngeren und den älteren Generationen. Martin Sökefeld geht davon aus, dass in Folge der alevitischen Bewegung in Deutschland erstmals eine Generation junger Alevitinnen und Aleviten auftrete, für die es normal sei, sich als Alevitin oder als Alevit zu bekennen und die nie die Erfahrung von takiye gemacht habe. ${ }^{1}$ Dagegen machte etwa Ingrid Pflüger-Schindbek 1989 während ih-

1 Vgl. Sökefeld 2008. Takiye bezeichnet »die Verheimlichung der eigenen religiösen Überzeugung in einer als feindlich wahrgenommenen Umwelt «. (Gorzewski 2010, S. 30.) 
rer Forschung zum Verhältnis junger Alevitinnen und Aleviten in Deutschland zu ihrem Glauben noch ganz andere Erfahrungen: Durch die Säkularisierung und die Ablehnung jeglicher religiöser Praxis seitens der Eltern sei damals eine religiöse Rückbesinnung der Kinder als eine mögliche Reaktionsform auf die veränderte Umwelt im Migrationskontext ausgeschlossen gewesen. ${ }^{2}$

In dem vorliegenden Beitrag wird jedoch gezeigt, dass heute durchaus gegenteilige Tendenzen erkennbar sind. Er will zudem Taşçıs These widerlegen, dass sich die junge Generation der Alevitinnen und Aleviten in Deutschland in "gravierenden Auflösungsprozessen « befinde und die alevitische Gemeinschaft lediglich durch die Abgrenzung zur sunnitischen Gemeinschaft definiert werden könne. ${ }^{3}$ Der Beitrag beleuchtet stattdessen, dass das Verhältnis zwischen Sunniten- und Alevitentum für viele Alevitinnen und Aleviten nach wie vor als speziell bis problematisch wahrgenommen wird. Daran knüpft sich die Frage an, ob junge Alevitinnen und Aleviten das Alevitentum im Islam verorten. Die divergierenden Verhältnisbestimmungen zum Islam analysierte Gorzewski und nannte als einen Grund für die Verortung des Alevitentums außerhalb des Islams, dass der islamische Einfluss auf das Alevitentum lediglich als äußerer Zwang im Rahmen der takiye bestehe, aber kein tatsächlicher Bestandteil des Alevitentums sei. Zudem gehe die voranschreitende Etablierung des Alevitentums in Deutschland mit einer Transformation überlieferter Traditionen und Rituale und deren Anpassung an einen deutschen Kontext einher. ${ }^{4}$ In diesem Zusammenhang warnt Vorhoff vor vorschnellen Veränderungen. Die fehlende Schrifttradition führe dazu, dass während der nunmehr erfolgenden Verschriftlichung viele unreflektierte und unterschiedliche Interpretationen des Alevitentums festgehalten würden. ${ }^{5}$ Inwieweit diese Voraussetzung eine aktuelle Herausforderung für alevitische Jugendliche darstellt, wird in dem Beitrag erläutert. Der Bildungsbeauftragte der AABF, Ismail Kaplan, geht allerdings davon aus, dass sich die alevitische Jugend in einer besseren Ausgangssituation für eine reflektierte Auseinandersetzung mit dem Alevitentum befinde als die älteren Generationen. Denn die Jüngeren seien nicht so sehr mit Vorurteilen und Repressalien seitens sunnitischer Radikaler belastet. ${ }^{6}$ Demnach wird im Folgenden auch gezeigt, inwiefern diese Ausgangssituation mehr Zugangsmöglichkeiten zu Informationen über das Alevitentum verschafft. Hinzu kommt außerdem die Tatsache, dass die jüngere Generation andere Medien wie zum Beispiel das Internet verstärkt nutzt. Can etwa

\footnotetext{
2 Vgl. Pflüger-Schindbek 1989.

3 Vgl. Taşçı 2008.

4 Vgl. Gorzewski 2010.

5 Vgl. Vorhoff 2000.

6 Vgl. Kaplan 2004.
} 
zeigt, dass das Internet für die junge Generation eine alternative Informationsquelle bietet, gerade dann, wenn durch die Eltern keine alevitischen Inhalte vermittelt wurden. Er beschreibt außerdem, wie die Identifizierung mit dem Alevitentum für Jugendliche das Bedürfnis nach partikulärer Identität stillt. ${ }^{7}$ Dieser Punkt spielt auch eine wichtige Rolle für die interviewten Jugendlichen und wird in diesem Beitrag analysiert.

Die oben erwähnten Fragen werden unter den Jugendlichen kontrovers und intensiv diskutiert und ihre Standpunkte wurden bisher kaum erforscht. Durch narrative Interviews kann direkt in Erfahrung gebracht werden, wie sich die Jugendlichen einordnen und wo sie sich von den älteren Generationen abgrenzen. Daher bilden vier narrative Interviews mit Studentinnen und Studenten aus Marburg zwischen 20 und 30 Jahren sowie ein Experteninterview mit dem Vorstandsvorsitzenden des BDAJ die Grundlage dieses Forschungsbeitrags. ${ }^{8}$ Zudem wurden Online-Informationen von der Homepage des BDAJ, von der AABF herausgegebene Literatur sowie die vorhandene Fachliteratur hinzugezogen. Dementsprechend orientiert sich die Forschungsarbeit an der "Objektiven Hermeneutik « Oevermannns mit » offenen «, gesprächsförmigen Interviews. ${ }^{9}$ Das Ziel dieser Methode ist nicht, "genaue Informationen « von dem Probanden oder einen $\mathrm{Zu}$ gang zum »subjektiven Standpunkt « zu erhalten, sondern »ein möglichst unverstelltes Protokoll einer sozialen Praxis jenseits der Interviewpraxis zu erhalten «. ${ }^{10}$ Zum anderen orientiert sich die Forschungsarbeit an der Methode nach SchmidtLauber, die den Schwerpunkt des qualitativen Interviews auf die »Kunst des Reden-Lassens « legt. Idealtypischerweise wird hierbei in einem offenen Gespräch die oder der Interviewte durch »erzählgenerierende Impulse « dazu angeregt, in einen freien Erzählfluss zu gelangen. ${ }^{11}$

Die Auswertung der Interviews wurde auf zwei Ebenen vorgenommen. $\mathrm{Zu}$ nächst wurden die Daten innerhalb der qualitativen Forschung anhand der »Grounded Theory « analysiert. Hierbei wird durch das ständige Vergleichen von Vorkommnissen innerhalb der Daten eine Generierung von theoretischen Eigenschaften vorgenommen. Dadurch werden aus den Daten Kategorien erstellt, die Antworten auf die Kernfrage nach den Unterschieden zwischen jüngeren und älteren Alevitinnen und Aleviten in Deutschland liefern. ${ }^{12}$ Für die anschließende Interpretation orientiert sich die Forschungsarbeit wiederum an einer zentralen

7 Vgl. Can 2008.

8 Die Interviews wurden im November und Dezember 2010 in Marburg und das Experteninterview mit Serdar Akin telefonisch geführt.

9 Vgl. Oevermannn et al. 1979.

10 Wernet 2000, S. 58.

11 Schmidt-Lauber 2007, S. 174.

12 Vgl. Strübing 2004, S. 13. 
forschungslogischen Ausrichtung der »Objektiven Hermeneutik «, die die latente Sinnstruktur und damit die soziale Praxis hinter der subjektiv-intentionalen Repräsentanz ermittelt. ${ }^{13}$

\section{Das Alevitentum in der Türkei und in Deutschland - Unterdrückung und die alevitische Bewegung}

Ihren Namen und die Entstehung des Alevitentums führen Alevitinnen und Aleviten auf Ali, den Schwiegersohn und Cousin des Propheten Mohammed, zurück, den sie als rechtmäßigen Nachfolger des Propheten sehen. ${ }^{14}$ Die Grundlage für das spätere Alevitentum bildeten allerdings Bewegungen, die bereits auf vorislamische Zeit zurückgehen. ${ }^{15}$ Jahrhundertelang hatten die Alevitinnen und Aleviten als überwiegend separate Glaubens-, Kultur- und Sozialgemeinschaft in Ostanatolien gelebt, standen oft zwischen den osmanisch-sunnitischen und iranisch-schiitischen Fronten oder wurden von der einen oder anderen Seite instrumentalisiert. Gerade in der spätosmanischen und frührepublikanischen Zeit waren sie als religiöse Minderheit Gegenstand von Unterdrückung und Vertreibung. ${ }^{16}$ Durch die Praxis der takiye und durch die soziokulturellen Umwälzungen infolge der sich in den 196oer Jahren verstärkenden Urbanisierung konnten wesentliche Bestandteile der religiösen Tradition nicht erhalten werden. Zur gleichen Zeit orientierten sich die Alevitinnen und Aleviten immer mehr an linksgerichteten Organisationen, wodurch eine Verschiebung vom religiösen Glauben zu säkularer bis anti-religiöser, linksorientierter Politik stattfand. ${ }^{17}$

Im Zuge der Anwerbeabkommen Deutschlands mit der Türkei kamen seit den 196oer Jahren viele Alevitinnen und Aleviten in die Bundesrepublik, in der heute schätzungsweise zwischen 500 ooo und 700 ooo Alevitinnen und Aleviten leben. ${ }^{18}$ Parallel zur alevitischen Bewegung in der Türkei, dessen Anhänger Anerkennung forderten, begann Ende der 1980er Jahre eine alevitische Bewegung in Deutschland. Nachdem die Alevitinnen und Aleviten sich lange bedeckt gehalten hatten, traten sie erstmals öffentlich und selbstbewusst bei der Alevitischen Kulturwoche

13 Vgl. Wernet 2000, S. 18.

14 Vgl. Sökefeld 2008, S. 10.

15 Vgl. Gorzewski 2010, S. 26. Diese Bewegungen wurden unter anderem durch Einflüsse des Manichäismus, Zoroastrismus, Mazdakismus sowie auch des Christentums und des Buddhismus geprägt. Vgl. ebd.

16 Vgl. Engin, Franz 2001.

17 Vgl. Gorzewski 2010, S. 11.

18 Vgl. Bundesamt für Migration und Flüchtlinge 2009 bzw. Kaplan 2004, S. 14. 
im Oktober 1989 in Hamburg auf. Daraufhin folgte eine Reihe von Vereinsgründungen in der Bundesrepublik. ${ }^{19}$

Demgegenüber waren die Umstände für das alevitische "Comeback « in der Türkei wesentlich schlechter, denn die Unterdrückung der Alevitinnen und Aleviten setzte sich auf blutige Weise fort. ${ }^{20}$ Ein Brandanschlag auf ein Hotel in Sivas am 2. Juli 1993, bei dem über 30 Teilnehmerinnen und Teilnehmer eines alevitischen Kulturfestivals ums Leben kamen, ${ }^{21}$ löste einen großen Mobilisierungsschub für die alevitische Bewegung in Deutschland aus. In diesem Zusammenhang wurden auch die Vorläufer der heutigen AABF und des BDAJ gegründet. ${ }^{22}$ Im Zuge dieser Bewegung traten auch neue, teilweise rivalisierende Deutungen der alevitischen Lehre auf. ${ }^{23}$

\section{Die Arbeit des BDAJ - Rasante Entwicklungen und ambitionierte Ziele}

Der BDAJ Verein wurde als Jugendorganisation der AABF zunächst unter dem türkischen Namen Almanya Alevi Gençler Birliği (AAGB) gegründet. ${ }^{24}$ Den deutschen Namen trägt der Verband erst seit Oktober 2009. Laut des interviewten Bundesvorsitzenden Serdar Akin richtet sich der Verein vor allem an Alevitinnen und Aleviten bis einschließlich 27 Jahren. Die Vorstandsmitglieder dürfen

19 Vgl. Sökefeld 2008, S. 23.

20 Heute kämpfen Alevitinnen und Aleviten in der Türkei noch immer für ihre Gleichberechtigung. Die vielversprechend klingende Initiative »Alevitische Öffnung « (Alevi açlımı) der Regierung Erdoğans von 2010 entpuppte sich als eine weitere nutzlose Maßnahme des Staates, um sich »seine eigenen «, dem Staat gegenüber loyalen Alevitinnen und Aleviten zu kreieren. Vgl. Alemdar, Çorapçıŏlu 2011.

21 Vgl. Sökefeld 2008, S. 15.

22 Vgl. Sökefeld 2008, S. 26 f. Bedeutend für die Bekanntheit in Deutschland waren die Reaktionen von alevitischer Seite auf den am 23. Dezember 2007 von der ARD ausgestrahlten »Tatort«-Krimi »Wem Ehre gebührt«. Die Reproduktion des seit Jahrhunderten auf sunnitischer Seite verbreiteten Vorurteils, Alevitinnen und Aleviten würden inzestuöse Orgien feiern, löste Demonstrationen in Berlin und anderen Städten mit mindestens 20 ooo Teilnehmerinnen und Teilnehmern aus. Vgl. ebd., S. 7.

23 Vgl. Dressler 2002, S. 11. Das heutige Interpretationsspektrum des Alevitentums reicht von mystischer Gemeinschaft über den wahren Islam oder den türkischen Islam hinzu einer synkretistischen Glaubensform oder einer Philosophie. Deswegen kann nicht von dem einen Alevitentum gesprochen werden. Vgl. Gorzewski 2010.

24 Die folgende kurze Skizzierung des Vereins beruht auf Informationen des Experteninterviews mit dem seit Oktober 2010 amtierenden Bundesvorsitzenden Serdar Akin und der Vereins-Homepage. Die Informationen der Homepage können nur unter Berücksichtigung ihrer Subjektivität gedeutet werden, da sie lediglich eine Inszenierung derjenigen Aspekte ist, die die für die Homepage Verantwortlichen preisgeben möchten. 
bei ihrer Wahl höchstens 35 Jahre alt sein. ${ }^{25}$ Der Verein ist gemeinnützig und nach eigenen Angaben selbständig, aber als "Tochterorganisation « natürlich eng mit dem Erwachsenenverband verzahnt. So ist Serdar Akin in seiner Funktion als Vorsitzender der Alevitischen Jugend ebenfalls amtierendes Vorstandsmitglied der AABF.

Die in der Vereinssatzung enthaltenen Schwerpunkte sind der Erhalt und die Erforschung der alevitischen Lehre, der Einsatz für ein friedliches und gerechtes Zusammenleben, für Menschenrechte und Minderheiten, die Unterstützung von Jugendlichen, die Gleichstellung von Mann und Frau, Religionsfreiheit, die Integration ausländischer Jugendlicher, Umweltschutz sowie kritisches Denken und Handeln und die demokratische Mitgestaltung der Gesellschaft. Der Verein zählt 96 sogenannte Ortsjugenden als Voll- und weitere 41 Ortsjugenden als Anschlussmitglieder in den elf westdeutschen Bundesländern. Es gibt fünf Regionalverbände, die als verbindende Glieder zwischen Bundesvorstand und den Ortsjugenden gesehen werden sollen. ${ }^{26}$

Seit dem Vorstandswechsel 2008 hat sich der Verein deutlich vergrößert. Seitdem haben sich die Mitgliederzahlen des BDAJ von 10 ooo auf 33000 und der Jahreshaushalt von 10000 auf knapp eine Million Euro erhöht. ${ }^{27}$ Der Hauptgeschäftsstelle in Dortmund, die 2008 gegründet wurde, folgten kürzlich eine Geschäftsstelle in Frankfurt am Main sowie eine weitere in München. Es gibt mittlerweile neun hauptamtliche Mitarbeiterinnen und Mitarbeiter. ${ }^{28}$ Laut des Bundesvorsitzenden wurde mit der Mitgliedschaft im Deutschen Bundesjugendring $^{29}$ im Oktober 2011 eines der wichtigsten Ziele erreicht. Allerdings sieht er die nun größte Herausforderung des Vereins darin, möglichst viele weitere Mitglieder zu akquirieren:

»So gelingt es einfach mehr, das große Potenzial, das da ist, in die richtigen Wege zu kanalisieren. Ansonsten droht der Jugendverbandsarbeit irgendwann die Gefahr, einer starken Fluktuation ausgesetzt zu sein und heterogen sehr unterschiedlich stark ausgeprägt zu sein.»

Das Spektrum der Aktivitäten des Vereins ist sehr vielfältig. Der BDAJ bietet zahlreiche Seminare an, organisiert Versammlungen, Podiumsdiskussionen, kulturelle und Großveranstaltungen wie etwa Konzerte und führt Exkursionen durch. Der

25 Vgl. »Bund der Alevitischen Jugendlichen in Deutschland e. V.: Satzung «, \$4 Mitgliedschaft.

26 Vgl. die BDAJ-Homepage auf www.bdja.de. Zugegriffen: 14. August 2012.

27 Vgl. ebd.

28 Vgl. ebd.

29 Der Deutsche Bundesjugendring ist eine Arbeitsgemeinschaft von bundesweit tätigen Jugendverbänden und Landesjugendringen. 
Verband veröffentlicht regelmäßig Texte, Mitteilungen und Stellungnahmen, die sich mit dem Glauben, der Geschichte oder aktuellen Themen befassen. Neben dem Newsletter, der laut Serdar Akin zirka 5 ooo Jugendliche erreicht, gibt es seit 2012 eine eigene Verbandszeitschrift namens »PLURAL «. Die Zeitschrift erscheint jährlich und beinhaltet Beiträge zum Alevitentum und dem Verbandsleben mit seinen Entwicklungen, Aktivitäten und Vorhaben sowie Berichte der fünf Regionalverbände.

Der BDAJ gewinnt ständig neue Partnerorganisationen für die projektbezogene Zusammenarbeit dazu. Er steht unter anderem in engem Kontakt mit dem paritätischen Wohlfahrtsverband und dem Informations- und Dokumentationszentrum für Antirassismusarbeit (IDA e. V.), in dessen Vorstand der Jugendverband ebenfalls vertreten ist. Außerdem arbeitet der BDAJ mit der evangelischen und der katholischen Kirche zusammen. Nach Serdar Akins Aussage soll der Verein bundesweit möglichst bekannt und als zuverlässiger Ansprech- und Projektpartner gesehen werden.

\section{Die Interviewten und ihre Distanzierung zur Elterngeneration: Selbstbewusstsein, Informationsmöglichkeiten und neue Themen}

Im Folgenden werden die vier interviewten Alevitinnen und Aleviten kurz vorgestellt und ihre Positionen im Hinblick auf die eingangs erwähnten Forschungsfragen nach der eigenen Identität und dem alevitischen Glauben sowie dem Verhältnis zur Elterngeneration, zur Vereinsstruktur, den Sunnitinnen und Sunniten und dem Leben in Deutschland wiedergegeben. Dabei wird deutlich, dass die Einstellungen gegenüber dem Verein unter den Interviewten ebenso variieren wie ihre Interpretation vom Alevitentum.

$\mathrm{Cem}^{30}$ ist zur Zeit des Interviews 21 Jahre alt und Student. Alevit zu sein, bedeutet für ihn durch die Beachtung der grundlegenden alevitischen Inhalte zu innerer Ruhe zu gelangen und das humanistische Weltbild zu fördern. Er ist nicht Mitglied in dem Verein. Seine skeptische Haltung gegenüber der Vereinsarbeit erklärt er sich durch die jahrelange Tätigkeit seines Vaters im AABF, die ihm gezeigt habe, dass der Verein hauptsächlich Lobby- und Netzwerkarbeit betreibe.

Meltem, Cems Schwester, ist zur Zeit des Interviews 23 Jahre alt und ebenfalls Studentin. Alevitin zu sein bedeutet für sie, Gott in sich selbst zu suchen, auf das Herz zu hören und tolerant zu sein. Sie kann für sich dem Verein nicht viel abge- 
winnen - weshalb auch sie kein Mitglied ist - freut sich aber, dass er anderen eine Identifikationsmöglichkeit und Plattform bietet.

Esra ist zur Zeit des Interviews 22 Jahre alt und ebenfalls Studentin. Für Esra bedeutet Alevitin zu sein, ein mit der deutschen, westlichen Gesellschaft gut zu vereinbarendes Leben führen zu können. Sie ist seit 2010 im Bundesvorstand des BDAJ.

Hidir ist zur Zeit des Interviews 30 Jahre alt, Studentin und Simultanübersetzerin für Türkisch und Deutsch. Für Hidir ist das Alevitentum eine Religion und Lebensphilosophie, die ihr hilft mit den Menschen und der Umwelt umzugehen. Sie ist kein Mitglied im BDAJ, sympathisiert allerdings sehr mit dem Verein.

Alle vier Interviewten ${ }^{31}$ berichten davon, dass sie sich während ihrer Kindheit einerseits gefragt hätten, was es eigentlich bedeute, Alevitin oder Alevit zu sein. Andererseits hätten sie sich aufgrund negativer Reaktionen im Umfeld und ihrer Erziehung nicht getraut zu sagen, welcher Religion sie angehören. Zum Teil hätten sie es auch nicht wirklich gewusst. Alle treten heute allerdings als selbstbewusste Alevitinnen und Aleviten auf.

Nach Esra fängt die Auseinandersetzung mit der alevitischen Identifikation bei vielen an, sobald sie sich gegenüber einem sunnitischen Umfeld rechtfertigen müssen:

"... ich bin mir aber eigentlich auch ziemlich sicher, dass es von vielen so ist. [...] Dass man sich wirklich in jungen Jahren schon damit angefangen hat, sich damit zu beschäftigen, weil man halt direkt irgendwie damit konfrontiert wurde.»

Aufgrund von Mobbing-Erfahrungen von der fünften bis zur achten Klasse durch einen sunnitischen Klassenkameraden informierte sich Esra über ihren Glauben. Auf diese Weise habe sie ihrem Klassenkameraden schlagfertiger gegenübertreten können. Zuvor wusste sie nichts über das Alevitentum.

Meltem erzählt, dass sie sich bereits früh für ihren Glauben interessiert habe und mit 12 Jahren ein Referat über das Alevitentum gehalten habe. Mit 14 oder 15 Jahren habe eine türkische sunnitische Freundin ihr aufgrund ihrer Zugehörigkeit zum Alevitentum die Freundschaft gekündigt.

»Und ich bin eigentlich so sozialisiert worden, dass man erstmal nicht sagt, dass man Alevit ist. Mittlerweile ist mir das egal. Ich sag das immer im zweiten Satz: >Hallo, ich bin Meltem und ich bin Alevitin. Wenn du ein Problem damit hast, dann geh raus, und wenn nicht, dann bleib hier sitzen «.«

31 Wenn im Folgenden von den Interviewten gesprochen wird, ist Serdar Akin nicht miteinbezogen. 
Esra und Hidir haben beide mit etwa 13 Jahren gemerkt, dass sie anders seien als ihre sunnitischen Mitschülerinnen und Mitschüler. Auf Nachfragen hätten sie herausgefunden, dass sie Alevitinnen sind.

»Ich habe nur irgendwann in der Schule gemerkt, ich bin anders. Ich kann die Gebete nicht. Meine Mitschüler [...] sind in einer Koranschule und ich durfte nicht hin. « (Hidir)

Auch Cem schildert eine persönliche Veränderung hinsichtlich seiner Haltung zu seinem »Alevit-Sein «. Während er in jüngeren Jahren sein Gegenüber lediglich darüber aufgeklärt habe, dass er Alevit ist, geht er heute noch einen Schritt weiter. Wenn jemand seinen Glauben diskriminiert, zieht er die Lehren des Alevitentums als Argumentationshilfe heran, um seinen Gesprächspartner auf dessen Diskriminierung hinzuweisen: "Man spricht ja schließlich von Mensch zu Mensch und das ist ja auch der alevitische Gedanke [...] «. Hieran zeigt sich, dass Cem so überzeugt von seinem Glauben ist, dass er andere bewusst davon in Kenntnis setzen möchte. Diese offensive Haltung zeugt von einer Loslösung von der takiye. Nach Kaplan könnten die alevitischen Jugendlichen »ein objektiveres und offeneres Verhältnis zum eigenen Glauben aufbauen als ihre Eltern «, da sie »bei ihrer Identitätsfindung nicht gezwungen « seien, » Maßstäbe aus dem Land ihrer Eltern und Großeltern, der Türkei, zu berücksichtigen «. ${ }^{32}$

Die eigene Einordnung des Alevitentums in Bezug auf den Islam zeigt ebenfalls, dass eine Distanzierung der jüngeren Alevitinnen und Aleviten von den älteren stattgefunden hat. Nach Kaplan lässt sich dies folgendermaßen erklären:

»Die überwiegende Mehrheit der alevitischen Jugendlichen ist der Ansicht, dass das Alevitentum mit dem Islam wenig zu tun hat, weil die Aleviten die sogenannten > Fünf Säulen des Islam` nicht akzeptieren und praktizieren. [...] Ältere Aleviten sagen, dass sie die besten Muslime seien, weil nach ihrer Auffassung Allah, Mohammed und Ali die wichtigsten Grundlagen des Islam seien. ${ }^{33}$

Während die AABF »eine eindeutige Stellungnahme zu diesem Thema « vermeidet, ${ }^{34}$ da sie vermutlich Bedenken vor der Auseinandersetzung mit einigen Kreisen hat, positionieren sich die Jugendverbandsmitglieder größtenteils als Religionsgemeinschaft außerhalb des Islams. Die Jugendlichen können heute auf viel-

32 Kaplan 2004, S. 32.

33 Ebd., S. 34.

34 Gorzewski 2010, S. 274. 
fältige, wissenschaftliche Quellen zurückgreifen, die seit den 1980ern entstanden sind. Im Gegensatz zu ihren Eltern haben viele Alevitinnen und Aleviten heute die Möglichkeit, am alevitischen Religionsunterricht teilzunehmen. Neben mehreren Bundesländern, die alevitischen Religionsunterricht in der Grundschule anbieten, ist in Nordrhein-Westfalen seit 2012 auch der Unterricht an weiterführenden Schulen möglich geworden. Kaplan weist daraufhin, dass Alevitinnen und Aleviten sich außerdem mit Hilfe der elektronischen Medien besser vernetzen und organisieren können. ${ }^{35}$

Eine Interviewte betonte, dass die alevitischen »Traditionen " unter hiesigen Alevitinnen und Aleviten so große Beliebtheit erfahren, dass sie mittlerweile viel professioneller die Tänze und Instrumente beherrschten als Jugendliche in der Türkei. Im Gegensatz dazu lehnte ein anderer Student die Möglichkeit des »Importierens des anatolischen Glaubens « nach Deutschland gänzlich ab, worauf später noch weiter eingegangen wird.

Laut Serdar Akin sei es erst durch die Professionalisierung der verbandlichen Strukturen möglich geworden, solche Themen jugendgerecht aufzubereiten. Der Generationenunterschied spiegelt sich daher auch in den verschiedenen Inhalten und Arbeitsweisen des Jugend- und des Erwachsenenverbandes wider. Da der BDAJ der AABF untersteht, sind die Möglichkeiten der Selbstbestimmung zwar begrenzt. Trotzdem beeinflusst der Jugendverband durchaus immer mehr auch den Erwachsenenverband. Alle Interviewten haben von einem deutlichen inhaltlichen und strukturellen Unterschied gesprochen, den sie darauf zurückführen, dass die Mitglieder des BDAJ »Deutschland-sozialisiert « und die Mitglieder der AABF überwiegend »Türkei-sozialisiert « seien. Cem erklärt sich den Unterschied damit, dass die Älteren sich aufgrund von leidvollen Erfahrungen für das Alevitentum engagieren.

"Das ist etwas ganz anderes als wenn ich sage >Ich bin in einem Dorf aufgewachsen und habe ein paar Kühe gehabt, einen blauen Himmel und habe keine Gewalterfahrungen gemacht oder Ohnmacht erfahren «. Natürlich behandel' ich dann andere Themen als Menschen, die aus der Unterdrückung kommen.«

Hidir stellt das heutige Auftreten der Alevitinnen und Aleviten in der Öffentlichkeit dem in den 1990er Jahren gegenüber. Damals habe es zum Beispiel »wirklich Protest " gegen das Massaker in Sivas gegeben und man habe Anerkennung gefordert. Durch diese Vorarbeit können junge Leute ihre Position heute differenzierter sehen, wodurch sie sich mehr auf breitflächige Aufklärungs-, Bildungs-, 
Netzwerk- und Öffentlichkeitsarbeit und das Vorantreiben der Integration konzentrierten. Nach ihrer Meinung hätten die Jüngeren durch die deutsche Sozialisation heute die Möglichkeit, eine sehr gute Ausbildung zu erlangen. Dadurch seien sie gebildeter und fortschrittlicher als die Älteren und praktizierten eine ausgesprochen professionelle und erfolgreiche Jugendverbandsarbeit.

Meltem dagegen möchte die Älteren jedoch nicht als rückständig darstellen. Sie hätte Gegenteiliges bei der Rede eines dedes ${ }^{36}$ anlässlich der Jubiläumsfeier eines Kulturvereins gehört:

»Also der ist wirklich auf die Jugend und Bildung und Wissenschaft eingegangen. Und kam dann mit Albert Einstein und was weiß ich. Und war total >Vordenker-mäßig und der ist schon über 80.«

Esra betont, dass sich besonders mit dem Wechsel des Vorstands und der Wahl Ali Doğans zum BDAJ-Bundesvorsitzenden im Februar 2008 in dem Verein sehr viel getan und verbessert habe. Denn zu der Zeit habe der Aufbau professioneller Organisationsstrukturen angefangen. Während der BDAJ vor fünf Jahren eher einem Laien-Verein geglichen hätte, sei er heute ein Verein, der »immer wieder im Rampenlicht stehe«. Serdar Akin weist zudem darauf hin, dass die Inhalte der AABF immer stärker auch von dem BDAJ bestimmt würden. Auch Esra sagt, dass die Mitglieder des BDAJ versuchten »von unten quasi die Älteren zu verändern «, die stolz auf die Jüngeren seien und sagen, »dass sie sich von uns 'ne Scheibe abschneiden können «. Beispielsweise sei insbesondere aufgrund des Einsatzes des BDAJ 2011 die Frauenquote im AABF eingeführt worden. ${ }^{37}$

Andererseits profitiere der Jugendverband auch von dem Erwachsenenverband, indem er "Tipps, Vorschläge und Rückendeckung " erhalte. Serdar Akin spricht von der Möglichkeit der wechselseitigen Partizipation. Diese enge $\mathrm{Zu}$ sammenarbeit zwischen der AABF und dem BDAJ zeigt sich auch darin, dass der ehemalige Bundesvorsitzende des BDAJ, Ali Doğan, heute Generalsekretär der $\mathrm{AABF}$ ist.

Ein weiterer Unterschied zwischen der jüngeren und den älteren Generationen ist die Sprache. Nach Akin könnten viele jüngere Alevitinnen und Aleviten heute viel besser Deutsch als Türkisch oder Kurdisch, wie auch die Interviewten teilweise bestätigten. Dabei ermöglicht es die deutsche Sprache dem BDAJ, der ja auch einen deutschen Namen trägt, eine leichtere Zusammenarbeit mit anderen Vereinen und eine schnellere Öffnung zur deutschen Mehrheitsgesellschaft hin als

36 Der dede ist der traditionelle alevitische Geistliche und Ritualspezialist.

37 "31. 12. 2011 Neujahrsbrief 2012 des BDAJ Bundesvorsitzenden Serdar Akın «. 
dies etwa beim AABF der Fall gewesen ist. Durch die Benutzung der deutschen Sprache und die Abwendung vom Türkischen ist die Religion nicht mehr an eine spezifische Sprache gebunden. Damit bildet das Türkische ein weniger wichtiges Identitätskriterium des Alevitentums. Dies ist auch ein Vorteil für die Alevitinnen und Aleviten, die Kurdisch, Zazaki oder eine andere Sprache sprechen.

\section{Wozu die professionelle Organisierung einer Glaubensgemeinschaft?}

Unter den Jugendlichen stellt sich auch die Frage, warum sie sich als Glaubensgemeinschaft überhaupt professionell organisieren sollten oder warum nicht, so etwa Esra:

"Also warum sollte man sich organisieren, das ist ja auch immer so eine Frage. Warum überhaupt? Warum sollte ich als Alevitin? Mir geht es blendend in Deutschland. Denn den Glauben kann auch jeder für sich leben. Jeder kann immer schön an den Gottesdiensten teilnehmen. Klar irgendwelche Fragen, Probleme bezüglich der Religion werden natürlich auch vom BDAJ behandelt. «

Hinsichtlich der Bedeutung des Vereins für ihre Religiosität haben die Interviewten demnach unterschiedliche Ansichten, die auch aus ihren divergierenden Vorstellungen vom Alevitentum hervorgehen.

"Also es war alles bisher ganz gut zu vereinbaren mit einer westlichen Gesellschaft jetzt, in der ich lebe. Und das ist für mich eigentlich Alevitin sein. Also das ist etwas, was ich wirklich zu schätzen weiß.»

Cem dagegen teilt Esras Einschätzung nicht:

»Es wird immer schwieriger in dieser Welt, in der wir leben, in Europa, ... [...] Man versucht irgendwelche Automatiken zu entwickeln, durch die versucht wird, es dem Aleviten leichter zu machen, Alevit zu sein. [...] Das wird halt entfremdet, um sich dem Zeitgeschehen anzupassen. [...] Man versucht da irgendwelche Brücken zu bauen, die man gar nicht bauen sollte.«

Darin bestätigt sich die bereits zu Beginn angesprochene Problematik der plötzlichen Verschriftlichung und Veröffentlichung »eine(r) Kultur, die bisher vor allem mündlich und nur innerhalb der eigenen, durch soziale, geistige und Blutbande fest verankerte Gemeinschaft weitergegeben wurde, in einem relativ kurzen 
Zeitraum plötzlich verschriftlicht und nach außen getragen wird. $\aleph^{38}$ Dies erkennt auch Ali Doğan und schließt sich diesem Argument an:

»Viele Jugendliche sind auf der Suche nach einem Dogma, einer Richtlinie, die das selbstkritische und eigenständige Denken nicht mehr erforderlich machen. Die Gefahr, dass das verschriftlichte Progressive von heute womöglich das Altmodische und Überholte von morgen sein könnte, wird von weiten Teilen der Jugend verkannt. «39

Demnach stellt sich folgende Frage: Wie kann der Jugendverband zu dieser schwierigen Neubestimmung beitragen und wo zeigen sich seine Grenzen? Cems Meinung nach sollte ein alevitischer Verein die anatolische Dorfstruktur als Vorbild haben. Wenn er diese » Grundideen von Gemeinschaft « ganz außer Acht lasse, könne das Alevitentum »nur fernab von Spiritualität « fortbestehen. Demnach handele es sich bei dem BDAJ um "nichts anderes als bei den JuSos, die sich mit den Strukturen der SPD auseinandersetzen, um dann mit 30, 31 in das ältere Lager zu wechseln «. Dabei käme es hauptsächlich auf Mitgliederakquise an. Serdar Akin entgegnet, dass auch religiöse Themen erst durch die professionelle Verbandsarbeit in jugendgerechter Art und Weise möglich seien. Das Alevitentum sei außerdem, so Akin auf Anfrage, »kein Selbstzweck, sondern sehr eng mit politischen und sozialen Fragen verzahnt «. Ali Doğan äußert ebenso Bedenken gegen Cems Meinung. Als » besorgniserregend « empfinde er die »zunehmende Fixierung und eine ausschließliche Identifizierung über die Religion und den Glauben « und beobachte » eine Apolitisierung bei der Jugend « ${ }^{40}$

Wie für ihren Bruder sei für Meltem ihr persönliches Verständnis von Alevitentum nicht mit der Vereinsarbeit kompatibel, allerdings stelle der Verein für viele andere einen befriedigenden Zugang zum Glauben dar. Darüber hinaus sei für Cem die Vereinsarbeit notwendig, um Druck auf die türkische Regierung ausüben zu können, um »ähnlich friedliche Bedingungen wie in Deutschland « zu schaffen (Interview Cem). Für Meltem sei dies ein »Kampf in Anführungsstrichen «, den sie ihren unterdrückten »Ahnen auch schuldig « seien. Und auch für Esra sei die Arbeit im Verein wesentlich mehr als ein »Glaubensbekenntnis«, denn das könne »man ja auch für sich « sprechen.

38 Vorhoff 2000, S. 62.

39 Doğan 2008, S. 34.

40 Ebd. 


\section{Schwierige Auseinandersetzung mit dem Sunnitentum}

Durch die oben beschriebene Entwicklung der letzten Jahrzehnte werden die Alevitinnen und Aleviten immer mehr als eigene Gruppe wahrgenommen, die sich von der zahlenmäßig größten Glaubensgruppe unter den Menschen mit Migrationshintergrund in Deutschland, den Sunnitinnen und Sunniten ${ }^{41}$, unterscheidet. Historisch betrachtet hat sich das Alevitentum als religiöse Minderheit in der Türkei fast immer mit der Verhältnisbestimmung zum Sunnitentum auseinandersetzen müssen. Denn das staatliche Amt für religiöse Angelegenheiten (Diyanet İşleri Başkanlığı), welches das religiöse Leben in der Türkei reglementiert, erkennt nur das orthodoxe Sunnitentum als legale religiöse Konfession im Einklang mit der republikanisch-kemalistischen Ordnung an.

Das Verhältnis zwischen Aleviten- und Sunnitentum spielt auch für die Interviewten eine wichtige Rolle. Esra und Hidir heben dies gleich zu Beginn des Interviews hervor.

»Weil immer wenn ich diese paradoxe Lebensweise sehe von anderen Menschen, denke ich mir immer > Gott sei dank hab ich so einen Glauben, der so gut zu vereinbaren ist mit dem Alltag «•«(Esra)

»Alevitin zu sein ist, glaube ich, schon was anderes als Aussage, als wenn man jetzt zum Beispiel sagen würde, man ist Moslem.« (Hidir)

Im Gegensatz zu ihrem eigenen Leben beobachtet Esra bei sunnitischen Kommilitoninnen und Kommilitonen, dass es ihnen sehr schwer falle, ihre Religion zu praktizieren und gleichzeitig als "ganz normales Mädchen « leben zu können. Meltem sagt, dass alevitische Mädchen im Gegensatz zu einigen sunnitischen Mädchen »alles dürfen«. Hidir betont, dass sie aus Überzeugung handele und nicht, weil sie »dafür in der Hölle schmoren « oder im »Himmel verwöhnt « würde.

Wie bereits oben angesprochen, hatte bei Esra, Hidir und Meltem erst das "Othering" von sunnitischer Seite dazu geführt, dass sie sich ihrer alevitischen Identität bewusst geworden sind. Die Verhältnisbestimmung zum Sunnitentum ist daher insofern als Teil ihres Verständnisses von Alevitentum anzusehen und in ihnen erhalten geblieben. So überrascht es auch kaum, dass die Interviewten häufig ihre Positionen in Anlehnung an das Sunnitentum rechtfertigten. Beispielsweise erzählt Hidir, dass sie »die vielen Gebete gar nicht« könne, da sie nie die Koranschule besucht habe. Von welchen Gebeten spricht sie? Später sagt sie, sie könne

41 Innerhalb des Sunnitentums gibt es verschiedene Strömungen. Hier wird von dem eher orthodoxen Sunnitentum, welches vom türkischen Staat vertreten wird, gesprochen. 
»halt die türkischsprachigen, alevitischen Gebete«, die hörten sich aber »nicht wie ein Gebet « an. Darin zeigt sich, dass sie die sunnitischen Gebete als Norm voraussetzt und die alevitischen Gebete als von dieser Norm abweichend einstuft. Demnach hält sie es für nötig das Alevitentum zu erklären und zu rechtfertigen.

Wegen diesen Gefühls des »Sich-Rechtfertigen-Müssens « empfindet Cem es als äußerst schwierig, anderen von seinem Glauben zu erzählen. Religiosität werde seiner Meinung nach »immer mit den konventionellen Religiositäten der monotheistischen Religionen gleichgesetzt «. In den »Augen eines Sunniten oder Katholiken « sei er natürlich » ungläubig «, weil er » nicht zu gewissen Zeiten an gewissen Orten vorzufinden « sei.

Hidir ist der Meinung, dass Alevitinnen und Aleviten in Deutschland nach wie vor einer gewissen Diskriminierung von sunnitischer Seite ausgesetzt sind. In einigen Stadtteilen Deutschlands sei der gesellschaftliche Druck in einem Stadtteil oder in einer Nachbarschaft, der "mahalle baskısı «, immer noch dem in der Türkei sehr ähnlich. ${ }^{42}$ Dadurch hätte man es mit einem westlichen Lebensstil, zum Beispiel im Minirock oder ohne im Ramadan zu Fasten, sehr schwer. So sei nach Esras Meinung auch die Verortung des Alevitentums außerhalb des Islams in einigen muslimischen Kreisen »wirklich mutig « und man würde herausgefordert, seine Positionierung zu erklären:

»Dann heißt es direkt > warum, ihr glaubt doch auch an Ali und ihr macht doch auch dies und das und warum seid ihr denn keine Moslems «. Aber ich kenne eine Familie, die Aleviten sind und auch sagen, dass sie Moslems sind, warum denn?»

Die Tatsache, dass immer mehr Alevitinnen und Aleviten in Deutschland ihren Glauben außerhalb des Islams verorten, hat demnach zur Folge, dass der Islam nicht mehr als verbindendes Kriterium zwischen den beiden Glaubensrichtungen gesehen werden muss. Dies kann vorteilhaft für die Gläubigen in Deutschland sein. Alevitinnen und Aleviten, die Esras Haltung vertreten, können sich beispielsweise dem Vorwurf, der sunnitische Islam teile gewisse Werte wie die Gleichberechtigung von Frau und Mann wegen seiner Bindung an die Scharia nicht, entziehen. ${ }^{43}$ Hidir erklärt sich die ihrer Meinung nach bessere Angepasstheit der Alevitinnen und Aleviten in Deutschland gegenüber den Sunnitinnen und Sunniten durch ihr »sehr weltoffenes humanistisches Weltbild «.

Da Alevitinnen und Aleviten in Deutschland bislang meistens im Schatten der Sunnitinnen und Sunniten standen, erscheint es logisch, dass sie sich bewusst ab-

42 Zum Phänomen des »Nachbarschaftdrucks « (mahalle baskısl) in der Türkei vgl. Toprak et al. 2009.

43 Vgl. Gorzewski 2010, S. 272. 
zugrenzen versuchen. Die AABF stellt zum Beispiel in ihrer Satzung die "prekären Themen ", nämlich die Gleichberechtigung der Geschlechter, den Schutz der Glaubensfreiheit und Menschenrechte und die Treue zu den deutschen Gesetzen in den Vordergrund. ${ }^{44}$

Cem ist der Meinung, dass es eines der Ziele des Vereines sei, gegen die sunnitische Dominanz »ein bisschen dagegen zu drücken «. Den Interviewten war es allerdings sehr wichtig, sich gegenüber den Sunnitinnen und Sunniten nicht als »die Besseren « zu positionieren. Andererseits möchten sie jedoch auf signifikante Unterschiede hinweisen. Hierbei betonen sie auch, dass sie die Unterschiede vor allem am orthodoxen Sunnitentum festmachten und längst nicht alle Sunnitinnen und Sunniten einschließen würden.

Inwieweit Alevitinnen und Aleviten tatsächlich weniger Probleme mit den Integrationsanforderungen in Deutschland haben als Sunnitinnen und Sunniten, wurde bislang noch nicht wissenschaftlich erhoben. Allerdings gibt es einige Hinweise, die dafür sprechen. So konnten einige Studien nachweisen, dass Alevitinnen und Aleviten eine deutlich höhere Einbürgerungsquote in Deutschland aufweisen als die türkischstämmige Bevölkerung insgesamt. ${ }^{45}$

\section{Resümee}

In den letzten drei Jahrzehnten haben rasante Entwicklungen innerhalb des Alevitentums stattgefunden. Das Ergebnis dieser Entwicklungen ist das Erlangen von Selbstbewusstsein und Anerkennung. Damit einher geht aber auch die Frage nach der Gestaltung der aus dem veränderten Kontext resultierenden Neuformulierung der Glaubensgemeinschaft.

Die interviewten Jugendlichen befinden sich in der Situation, als junge Generation einerseits von den Entwicklungen profitieren zu können, andererseits auch Antworten auf die neuen Bedingungen geben zu müssen. Hierbei befinden sie sich in einer anderen Ausgangsposition als die Generation ihrer Eltern und Großeltern. Vor diesem Hintergrund setzen sie andere Akzente.

Bei den Interviewten hat ein Prozess stattgefunden, an dessen Anfang sie nichts über ihren Glauben wussten. Als Teenager haben sie sich dann zum Teil auf beschwerliche Weise mit dem Glauben auseinandergesetzt und schließlich haben sie ein selbstbewusstes Verhältnis zum Alevitentum entwickelt. Ihre heutige Haltung unterscheidet sich deutlich von der der älteren Generationen, die zum größ-

44 Vgl. »Alevitische Gemeinde Deutschland e. V.: Satzung «, \$ 2, Zweck und Ziele der AABF, sowie auch Sökefeld 2008, S. 272.

45 Vgl. Schmidt 2000, S. 86 ff., Sökefeld 2008, S. 20. 
ten Teil in der Öffentlichkeit nach wie vor unsicher zu ihrem Glauben stehen. Es ist zu vermuten, dass die Nachfolgegeneration der Interviewten gar keine persönlichen Erfahrungen mehr mit takiye machen.

Der für die jüngere Generation veränderte Zugang zu ihrem Glauben birgt zum einen sehr viel mehr Möglichkeiten. Durch mediale Vernetzung, zahlreiche Publikationen und die professionelle Vereinsarbeit können sich jugendliche Alevitinnen und Aleviten in Deutschland heute wesentlich vielfältiger informieren und ihren Glauben praktizieren, als die älteren es konnten. Zum anderen sind sie ebenso herausgefordert zu reflektieren, dass diese Vielfalt von Informationen vor dem Hintergrund eines bislang nicht existierenden Kanons auch zu Irritationen oder Unsicherheiten führen kann.

Da die AABF der Dachverband der Alevitinnen und Aleviten in Deutschland ist, spielt sie bei den Entwicklungen des Alevitentums eine maßgebliche Rolle. Doch der Jugendverband nimmt sukzessive eine ebenfalls sehr bedeutende Position ein. Durch den offiziellen Status, den der Verein durch die Aufnahme in den Deutschen Bundesjugendring 2011 erhalten hat, hat er mehr Handlungsspielraum erhalten und kann sich besser vernetzen. Als Vertreter von 33 ooo jugendlichen Alevitinnen und Aleviten deutschlandweit kann der Verein sich als größte so genannte Selbstorganisation von jugendlichen Migrantinnen und Migranten bezeichnen. Ausschlaggebend im Vergleich zum AABF ist jedoch die Tatsache, dass der Migrationshintergrund im BDAJ immer mehr an Bedeutung verliert. Dadurch, dass die Mitglieder in Deutschland sozialisiert sind, fokussieren sie ihre Aktivitäten auf Deutschland. Sie bringen damit das Alevitentum auf eine Ebene, die immer losgelöster von der Türkei ist.

Auch die derzeitige Etablierung des alevitischen Religionsunterrichts hat bedeutende Auswirkungen auf das Alevitentum in Deutschland. Wenn der Unterricht bald in weiteren Bundesländern eingeführt und auch an immer mehr Schulen tatsächlich durchgeführt wird, könnten immer mehr Kinder und Jugendliche bereits in der Schule einen fundierten Zugang zu ihrem Glauben sowie einen selbstverständlicheren Umgang mit diesem erhalten.

Durch das selbstbewusste Auftreten der Jugendlichen und ihrer Betonung der Eigenständigkeit und der unter ihnen weit verbreiteten Auffassung der Nicht-Zugehörigkeit zum Islam können sie es schaffen, als eigene Gruppe von der Mehrheitsgesellschaft wahrgenommen zu werden. Dies bedeutet einerseits, dass sie sich nicht ständig erklären und rechtfertigen müssen und anerkannt sind. Andererseits können sie einen Beitrag dazu leisten, den bestehenden Diskurs über »den Islam « als eine homogene Einheit zu verändern und auf die Vielfältigkeit unter den Menschen mit Migrationshintergrund hinzuweisen.

So haben die jungen Alevitinnen und Aleviten in Deutschland viele Möglichkeiten, stehen aber auch vor vielen Herausforderungen, allen voran ihrem im- 
mer noch angespannten Verhältnis mit der sunnitisch-orthodoxen türkischen Mehrheit unter den Menschen mit Migrationshintergrund in Deutschland. Neben den Themen, die in dem Artikel behandelt wurden, gibt es viele zusätzliche interessante Aspekte hinsichtlich der weiteren Entwicklung des Alevitentums in Deutschland, wie etwa die Frage des Blutrechts oder die Frage nach der Gottesdienstgestaltung.

\section{Quellen- und Literaturverzeichnis}

Alemdar, Z., Çorapçığlu, R. B. 2011. Alevis and the Turkish State. Turkish Policy Quarterly $10(4), 117-124$.

Can, H. 2008. Außenseiter wider Willen: Das >coming-out ‘ des Alevitentums in der diasporischen Enkelgeneration oder Erinnerungs- und Identitätsarbeit über das digitale Gedächtnis des Internets. In Aleviten in Deutschland - Identitätsprozesse einer Religionsgemeinschaft in der Diaspora, hrsg. M. Sökefeld, 155-194. Bielefeld: Transcript.

Doğan, A. 2008. Alevitische Jugend - Quo Vadis?. Wo bewegt sich/Wohin muss sich die alevitische Jugend hinbewegen? Alevilerin Sesi 116: 34-35.

Dressler, M. 2002. Die Alevitische Religion: Traditionslinien und Neubestimmungen. Würzburg: Ergon.

Engin, İ., Franz, E. (Hrsg.) 2001. Aleviler/Alewiten. Cilt/Band 1: Kimlik ve Tarih/Identität und Geschichte. Hamburg: Deutsches Orient-Institut.

Gorzewski, A. 2010. Das Alevitentum in seinen divergierenden Verhältnisbestimmungen zum Islam. Berlin: EB-Verlag.

Haug, S., Müssig, S., Stichs, A. 2009. Muslimisches Leben in Deutschland. Forschungsbericht im Auftrag der Deutschen Islam Konferenz 6, hrsg. Bundesamt für Migration und Flüchtlinge. Nürnberg.

Kaplan, I. 2004. Das Alevitentum - Eine Glaubens- und Lebensgemeinschaft in Deutschland. Köln: Alevitische Gemeinde Deutschland e. V.

Oevermann, U., Allert, T., Konau, E., Krambeck, J. 1979. Die Methodologie einer >objektiven Hermeneutik und ihre allgemeine forschungslogische Bedeutung in den Sozialwissenschaften. In Interpretative Verfahren in den Sozial- und Textwissenschaften, hrsg. H.-G. Soeffner, 352-434. Stuttgart: Metzler.

Pflüger-Schindbek, I. 1989. Achte die Älteren, liebe die Jüngeren: Sozialisation türkisch-alevitischer Kinder im Heimatland und in der Migration. Frankfurt am Main: Althenäum.

Schmidt, S. 200o. Kurdisch-Sein, mit deutschem Pass. Formale Integration, kulturelle Identität und lebensweltliche Bezüge von Jugendlichen kurdischer Herkunft in NordrheinWestfalen: eine quantitative Studie. Bonn: Navend Zentrum für Kurdische Studien.

Schmidt-Lauber, B. 2007. Das qualitative Interview oder: Die Kunst des Reden-Lassens. In Methoden der Volkskunde. Positionen, Quellen, Arbeitsweisen der Europäischen Ethnologie, hrsg. S. Göttsch, A. Lehmann, 169-188. Berlin: Reimer Verlag.

Sökefeld, M. 2008. Einleitung: Aleviten in Deutschland - von takiye zur alevitischen Bewegung. In Aleviten in Deutschland - Identitätsprozesse einer Religionsgemeinschaft in der Diaspora, hrsg. M. Sökefeld, 7-36. Bielefeld: Transcript. 
Strübing, J. 2004. Grounded Theory. Zur sozialtheoretischen und epistemologischen Fundierung des Verfahrens der empirisch begründeten Theoriebildung (Qualitative Sozialforschung). Wiesbaden: VS.

Taşçı, H. 2008. Die zweite Generation der Alevitinnen und Aleviten zwischen religiösen Auflösungstendenzen und sprachlichen Differenzierungsprozessen. In Aleviten in Deutschland - Identitätsprozesse einer Religionsgemeinschaft in der Diaspora, hrsg. M. Sökefeld, 133-154. Bielefeld: Transcript.

Toprak, B., Bozan, İ., Morgül, T., Şener, N. 2009. Being different in Turkey - Religion, Conservatism and Otherization. Research Report on Neighbourhood Pressure. Istanbul: Boğaziçi University, Open Society Foundation Turkey.

Vorhoff, K. 200o. Alevitische Identität in der Türkei heute. In Aleviler/Alewiten. Cilt/Band 1: Kimlik ve Tarih/Identität und Geschichte, hrsg. I. Engin, E. Franz, 59-74. Hamburg: Deutsches Orient-Institut.

Wernet, A. 2000. Einführung in die Interpretationstechnik der Objektiven Hermeneutik. Opladen: Leske und Budrich.

\section{Internetquellen}

"31. 12. 2011 Neujahrsbrief 2012 des BDAJ Bundesvorsitzenden Serdar Akın «. www.aagb.net/ index.php?option=com_content\&view $=$ article $\&$ id $=407$ :neujahrsbrief-2012-desbdaj-bundesvorsitzenden-serdar-akn\&catid=65:pressemitteilungen \&Itemid=225. Zugegriffen: 8. August 2012.

"Alevitische Gemeinde Deutschland e. V.: Satzung «, www.alevi.com/de/wir-uber-uns/satzung/. Zugegriffen: 14. August 2012.

»Bund der Alevitischen Jugendlichen in Deutschland e. V.: Satzung «. www.bdaj.net/PDFDaten/satzung-bdaj.pdf. Zugegriffen: 14. August 2012.

Open Access. This chapter is distributed under the terms of the Creative Commons Attribution Noncommercial License, which permits any noncommercial use, distribution, and reproduction in any medium, provided the original author(s) and source are credited. 